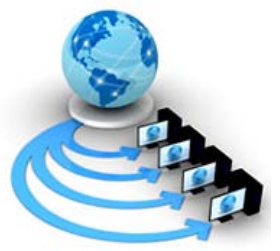

Volume 9, No. 3, May-June 2018

\title{
SPAM DETECTION FRAMEWORK USING SENTIMENATL ANALYSIS
}

\author{
Vibha Vinod \\ Master Student \\ Dept. of Computer Science and Engineering \\ BMSIT \& M, Bengaluru,Bangalore, India
}

\author{
Durgadevi G.Y \\ Assistant Professor \\ Dept. of Computer Science and Engineering \\ BMSIT\&M Bangalore, India
}

\begin{abstract}
In this technically knowledgeable world, conclusions and surveys available to us are a standout amongst the most unequivocal and requesting factors in building up our perspectives and affecting the accomplishment of a brand, item or service. With the entry and development of online networking, associates frequently express their feelings on prevalent web-based social networking stages. The likelihood that anyone can leave an audit makes a susceptible door for spammers to compose spam surveys about items, administrations and services. Recognizing the spam substance will be the primary target of the proposed spam recognition system. Once the spam audits are sifted through from the unsupervised informational index, whatever remains of the surveys will shape regulated informational collection which forms the supervised information on to which sentimental analysis approach will be applied and calculations are done, so as to gauge the assumption estimation of each audit based on the opinion value found
\end{abstract}

Keywords: Sentimental, spam, behaviour, spammer, reviews

\section{INTRODUCTION}

Web mining is the use of information mining strategies to recognize designs from the World Wide Web which includes Online Social Media that structures a piece of Web 2.0. The data about the examples found on social entries can be congregated by mining the web, which can be accomplished by using the idea of Sentimental Analysis in this way offering ascend to another time of Web Intelligence.

Online gateways found on the web, accept a mind-boggling part in information raising which is believed to be a crucial wellspring of getting commonness for an alternate extent of endeavors, for instance, design, social protection, preparing, friendliness, cultivating et cetera. These sections go about as a center man to advance things from these endeavors and goes about as an overall mediator to connect with customers to give the best idea of organizations. It is seen, from the past couple of year's kinfolk who routinely purchase things or organizations from these online social doors settle on decisions in light of the made studies which empowers or cripples them in their assurance of things and organizations. [1]

Then again, a lot of writing has been distributed on the systems used to recognize spam and spammers and in addition distinctive sort of investigation on this theme. These systems can be classfied into various classifications; some utilizing phonetic examples in content, which are for the most part in view of bigram, and unigram [2], others depend on behavioral examples that depend on highlights removed from designs in clients' conduct which are generally metadata based, and even a few procedures utilizing diagrams and chart based calculations and classifiers [3].

The way that anyone with a character winds up endorsed to comment a review, makes an alluring open entryway for spammers to make fake studies arranged particularly to mislead customer's inclination. The effect of such spam comments can be restricted or wiped out by joining the thoughts of Web Content and Structure Mining close by the likelihood of Classification and Clustering found in insightful examination strategy which is seen in nostalgic thinking.

\section{LITERATURE SURVEY}

Spam is everywhere throughout the web. From your inbox to your blog remarks, it's probable that you experience spam regularly. Also, now there's another zone of spam entrepreneurs should know about - audit spam [10].

Spam surveys are audits that are completely or mostly false, or that breaks the rules of the site on which the audit shows up.

Extensively, audit spam falls into two classifications. The first is individuals who are attempting to influence their own business to look great. The second is individuals who are endeavoring to harm another person's business. The inspiration for the principal gathering of individuals is quite evident they either need to influence their business to seem better known, or they need to their business to rank higher in survey site indexed lists.

Essentially three sorts of audit spams exist [4]. These are:

Sort 1 (Untruthful Review Spams): Fictitious positive surveys are remunerated to items with a specific end goal to advance them and furthermore preposterous negative surveys are given to the contending items to hurt their notorieties among the customers. This is how untruthful surveys delude the customers into trusting their spam audits. Sort 2 (Reviews with mark specifies): These spams have just brands as their prime core interest. They remark about the producer or merchant or the brand name alone. These surveys are one-sided and can without much of a stretch b figured out as they don't talk about the item and rather just say the brand names. 
Sort 3 (Non-audits): These surveys are either garbage, as in, have no connection with the item or are absolutely utilized for ad purposes.

In the most recent decade, an awesome number of research considers center around the issue of spotting spammers and spam audits. Nonetheless, since the issue is noninsignificant and testing, it stays a long way from completely explained. We can abridge our talk about past examinations in three after classifications.

\section{A. Semantic based Methods}

This approach extricate semantic based highlightsfinod spam audits. Feng et al. utilize unigram, bigram and their organization. Different examinations utilize different highlights like pairwise highlights formding spam audits [5].

\section{B. Behavioural based Methods}

Jindal et. al extricate 36 behavioral highlights and utilize a managed strategy fond spammers on Amazon and demonstrates behavioral highlights demonstrate spammers' character superior to phonetic ones [6].

\section{Graph Based Method}

Wang et al [7]. Likewise make a system of clients, audits and things and utilize essential suppositions (for instance an analyst is more dependable on the off chance that he/she composes more genuine reviews) and mark surveys. Wahyuni [8] proposes a half breed strategy for spam discovery utilizing a calculation called $\mathrm{ICF}++$ which is an augmentation to ICF of in which simply audit rating are utilized tofind spam identification. This work utilize likewise opinion investigation to accomplish better precision specifically

\section{PROPOSED METHODOLOGY}

The thought behind the proposed framework is to utilize a novel spam acknowledgment framework which utilizes metapath methods to distinguish spam content $\mathrm{n}$ spammers on the web and furthermore another outline based procedure to check behavioural estimation of each substance.

The surveys composed on social entries if hopeful in nature bring benefit and notoriety for the business and if negative in nature annihilates the brand name of the business. This gives spammers an opportunity to post a manufactured survey. Uncalled for positive audits and unmerited negative surveys are utilized by the majority of the spammer groups and people. It is seen, there exists still a substantial piece of the group who are new of such spamming strategies and such counterfeit and one-sided surveys. Subsequently there is a requirement for an impartial spam location system to sift through beguiling remarks from the spammer. In this way, giving an impartial sentiment to all clients of an item or benefit and consequently keeping the destruction of bona fide business.

The main specification of proposed framework are as follows:

1. It can be exceptionally viable in distinguishing spam surveys.

\section{It prompts a superior execution.}

Important definitons to keep in mind to understand propsoed framework are as follows:

1) Heterogeneous Information Network- Assume we have $r(>1)$ sorts of hubs and $s(>1)$ kinds of connection interfaces between the hubs, at that point a heterogeneous data arrange is feed as a diagram $\mathrm{G}=(\mathrm{V}, \mathrm{E})$ where eachnode $\mathrm{v} \in \mathrm{V}$ andeachlink e $\in$ E belongstooneparticular hub compose and connect type individually. On the off chance that two connections have a place with a similar kind, the sorts of beginning hub and closure hub of those connections are the same [1].

2) Metapath [1]- As specified above, there are no edges between two hubs of a similar kind, however there are ways. Given a heterogeneous data arrange $G=(V, E)$, a metapath $P$ is dened by a succession of relations in the system blueprint $\mathrm{T}=(\mathrm{A}, \mathrm{R})$, indicated in the frame A1(R1)A2(R2)...(R(l-1))Al, which defines a composite connection $\mathrm{P}=\mathrm{R} 1 \mathrm{R} 2$...R $(\mathrm{l}-1)$ between two hubs, where $\mathrm{o}$ is the sythesis administrator on relations. For accommodation, a metapath can be spoken to by an arrangement of hub writes when there is no equivocalness, i.e., $\mathrm{P}=\mathrm{A} 1 \mathrm{~A} 2$...Al. The metapath expands the idea of connection writes to way composes and portrays the diverse relations among hub writes through circuitous connections, i.e. ways, and furthermore infers assorted semantics.

3) Network schema [1]- Given a heterogeneous data arrange $G=(V, E)$, a system pattern $T=(A, R)$ is a metapath with the protest write mapping $\tau: \mathrm{V} \rightarrow$ An and interface mapping $\varphi: \mathrm{E} \rightarrow \mathrm{R}$, which is a chart deined over question compose $\mathrm{A}$, with joins as relations from $\mathrm{R}$. The construction depicts the metastructure of a given system (i.e., what number of hub writes there are and where the conceivable connections exist

\section{SYSTEM DESIGN}

The figure 1 below shows the architecture of proposed spam detection framework.

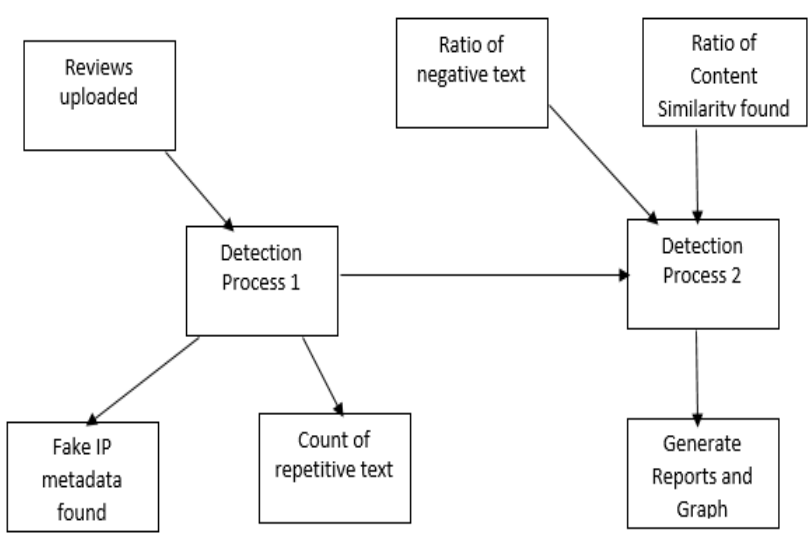

Figure 1: System Architecture

It gives an outline of the framework design of the proposed spam location system. The audits are transferred onto the framework by means of Excel sheets, after effective transfer the surveys are handled by Detection Process 1 and 
Detection Process 2 to sift through the positive and negative writings. The two location forms use the already said calculations which are metapath based calculation to discover the way and network between the IP address from where the audits where transferred and the real substance being transferred. The second calculation basically centers on ordering the substance into the positive and negative classifications and acquiring the check of each dreary word and updates consider of the surveys often as possible by the recognized spammers. The above figure clarifies the framework engineering structure application perspective.

\section{Proposed Module Description}

Module 1- In the Upload Excel File Module, client needs to choose the record from the customer machine and the document substance will be sent to the server through URL as multi part, in the server side servlet gets the record content and compose the document content in the organizer of the application. From that envelope it peruses the document substance and store the record content in to the database.

\section{Module 2-Process 1 to identify counterfeit surveys:}

In the Fake Review Detection 1 process, Data will be perused from the database and checks whether the IP_Address and User ID is phony or not founded on the meta information table and embed the phony surveys in to the phony audit table. And furthermore it checks whether the quantity of surveys from the IP_Address are surpassing as far as possible with in the edge time restrict, if any IP_Address surpasses as far as possible, at that point that audits will be embedded to the phony audit table and that IP_Address will be embedded to the Meta Fake IP_Address table and rest of the surveys will be embedded to the Real audits table

\section{Module 3-Process 2 to identify counterfeit audits:}

In the Fake Review Detection 2 process, audits will be perused from the Real surveys table, considering every audit , in the main level, superfluous words and uncommon characters will be expelled, in the second level order every last word is thing or descriptor, in the third level paring the thing and nearby modifier in the fourth level checks whether the descriptive word which is matched with the thing is negative or positive, in the fifth level checks whether the greatest number of sets are certain or negative, in view of the most extreme tally of positive or negative, relegate the survey an incentive as positive or negative , in the 6th level compute and embed the two gram and three gram combines in to the database, in the seventh level ascertain the tally positive rate and n-gram level of every client and include every one of the rates and get add up to rate edge, if any client surpasses add up to rate limit, consider that client is phony and embed that client in to the meta counterfeit client table.

\section{Module 4-Report Generation:}

Report 1: will be created in light of the audits given by the particular client for the particular item.

Report 2: will be created in view of the surveys given by every one of the clients for the particular item.

Report 3: will be created in view of the audits given by the particular client for every one of the items.
Report 4: will be created in view of the phony surveys given by every one of the clients for every one of the items.

Report 5: will be created in light of the audits given by the Meta Fake clients and Meta Fake IP Address for every one of the items

\section{Module 5-Generating Graphs:}

Graph 1: is a Pie Chart, it will be created in light of the aggregate number of phony surveys, genuine audits and Meta counterfeit audits given by every one of the clients for every one of the items.

Graph 2: is a Bar Graph, it will be created in light of the quantity of surveys phony, genuine and Meta counterfeit audits V/s Products.

The Figure 2 gives a thought of the framework engineering found at the backend of the proposed venture. It is watched, the task takes after the Model View Controller Architecture which is an example utilized for creating applications in light of UIs. The accompanying advances are seen at the backend-

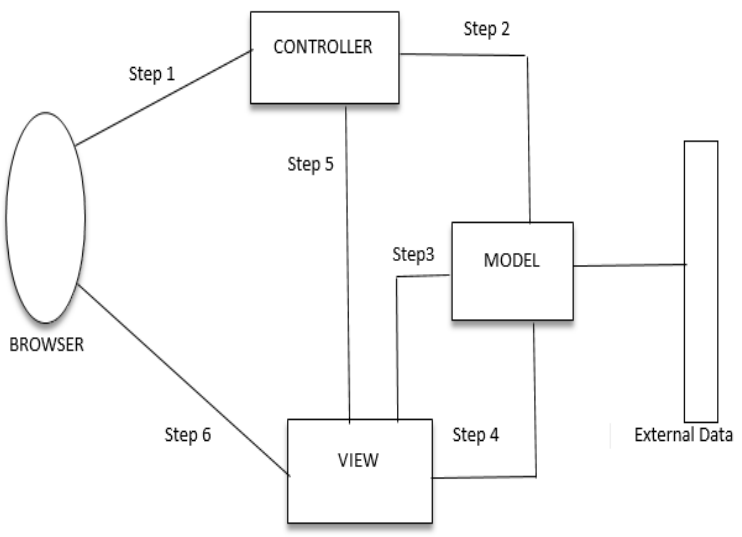

Figure 2- Backend design of system

Stage 1-User Requests are sent to the Controller from the program

Stage 2- Controller deals with the solicitations and select the right model and view, exchanges the solicitations to the model

Stage 3-Model performs epitome of demand, send this information to the view

Stage 4-The view readies the information, and sends back the refreshed demand to the model

Stage 5-Controller selects the ideal view for the coveted demand

Stage 6-View sends the last refreshed information as HTML substance to coordinate the client ask

The model gets to any outside wellspring of information to fulfill the client demands

\section{RESULTS AND DISCUSSIONS}

The results obtained for the proposed system is described below:

1. Admin successfully launches the services of the server, which can be accessed only by authorized user.

2. Admin successfully logins into the proposed system. 
3. Admin uploads the review dataset to be analyzed by training model.

4. Process 1 performs extraction of IP metadata and count of each text found in review set

5. Process 2 has three levels-

- At Level 1, unnecessary words are removed

- At Level 2, Pairing of words are performed

- $\quad$ At Level 3, Check is performed to see if pairs fall into category such as positive or negative reviews

6. Reports and graphs can be generated by admin based on criteria such as

- $\quad$ One user-One Product

- One product-All User

- One User-All Product

- Fake Reviews by users

- Meta fake reviews

- Meta real reviews

\section{List of Reports}

- One user - One Product

- One Product - All User

- One User - All Product

- Fake Reviews By all Users

- Meta Data Fake Review

- Meta Data Real Review

Figure 3- Choice of Reports being displayed

Figure 3 depicts the option of reports displayed to the user. The user role will be played by the admin, who can generate reports based on the options being displayed onto the screen. Each report option generates a list of information which shows the time and IP address from where the spam audit was posted. It also displays information if the User ID is genuine or not.

Figure 5- Bar grapgh of Experimental results on single product

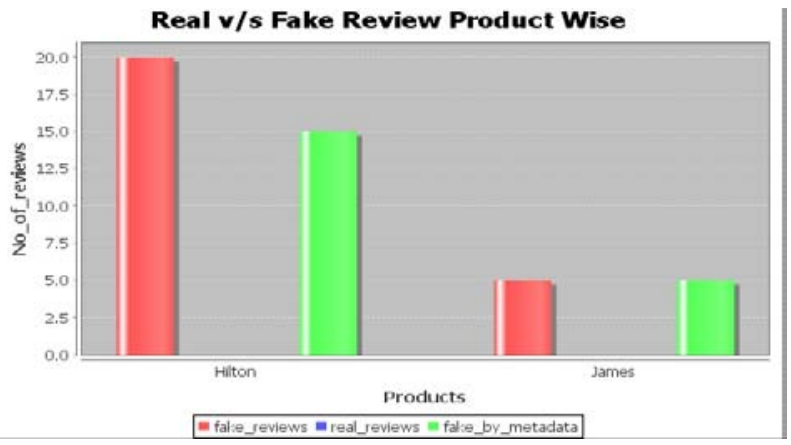

Figure 6- Bar graph of experimental results on two products

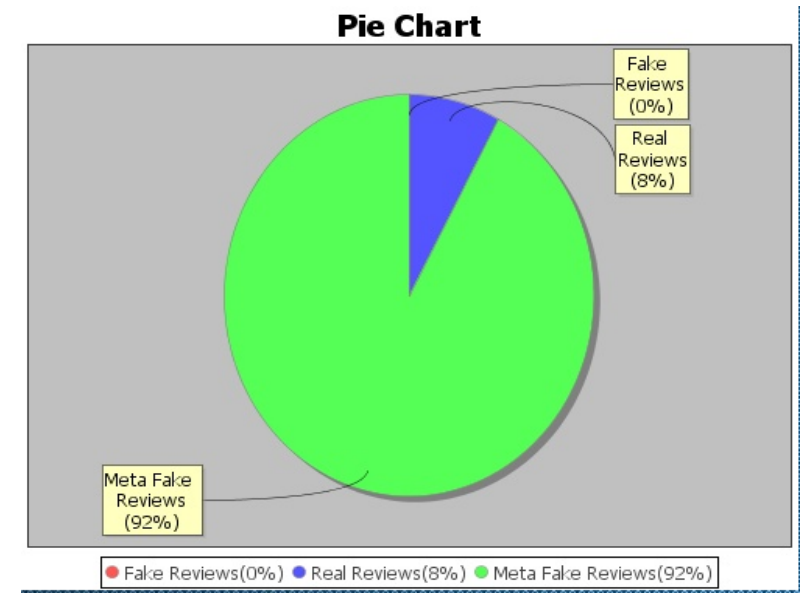

Figure 4- Pie Chart displayed for the reviews

Figure 4 displays a Pie chart of the reviews uploaded by the admin. This Pie chart gives an idea of how many audits i.e. reviews were fake or real in nature. From the experiment performed it was observed that one set of reviews uploaded yielded $8 \%$ of real reviews and the rest $92 \%$ of it were detected as fake by the detection framework. Different review sets will display different Pie charts based on the percentage of fake detected by proposed system.

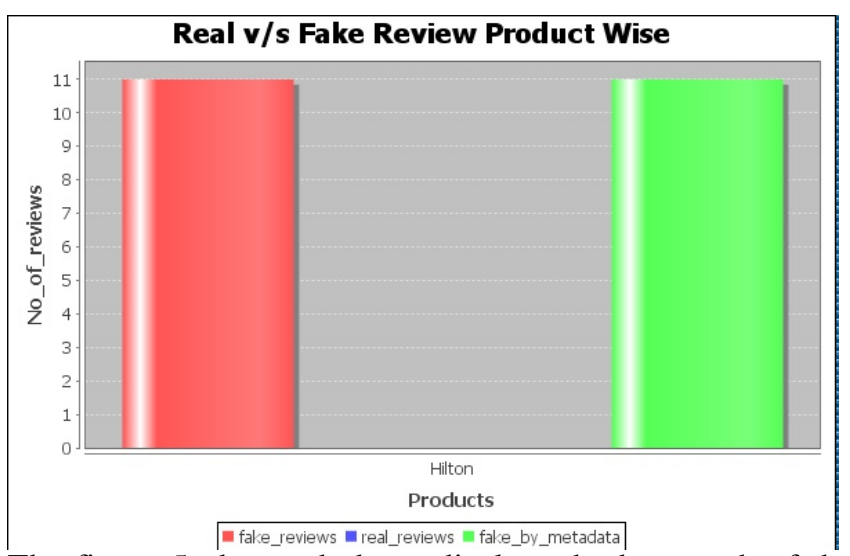

The figure 5 observed above displays the bar graph of the statistics found for the review set of a single product. The experiment was conducted for a single product named Hilton as a name for a hotel and its services. The review set for the product was found to be fake and its metadata was displayed

The figure 6 observed above displays the bar graph of the statistics found for the review set of a two products. The graph is generated based on the review set being uploaded onto the proposed system.

\section{CONCLUSION AND FUTURE SCOPE}

The proposed system influences the usage of a novel spam acknowledgment to structure in light of the metapath figuring to name reviews contingent upon a rank-based naming procedure. The execution of the proposed structure is evaluated by using review sets replicated by observing genuine study sets found on standard social doorways. The discernments exhibit that registered weights by using this metapath thought can be to a great degree fruitful in 
recognizing spam studies and prompts an unrivaled execution. Furthermore, in the wake offining four rule orders for features the recognitions exhibit that the reviews behavioral arrangement performs better than anything diverse orders, similar to zone under twist and furthermore in the found out weights. The results moreover confirm that using unmistakable supervisions, similar to the semi-regulated method, have no detectable effect on choosing a substantial part of the weighted features, likewise as in different datasets

For future work, metapath can be associated with various issues in this field. For example, practically identical structure can be used to find spammer gatherings. For finding gathering, reviews can be related through get-together spammer features and reviews with most raised equivalence in light of metapath thought are known as gatherings. Also, utilizing the thing incorporates is an interesting future work on this examination as it is used to distinguish spammers and spam studies. Unfortunately, in light of insurance concerns, we don't procure the customer information on the predetermined destinations and simply those locales can analyze the customer data inside. It will be conceivable to check for the true blue rest of the substance by planning the reviews with information open in the official locales for the given things. It is conceivable to use this proposed framework as a standard for helping updates in this investigation region.

\section{REFERENCES}

[1] Saeedreza Shehnepoor, Mostafa Salehi, Reza Farahbakhsh, Noel Crespi, "NetSpam: a Network-based Spam Detection Framework for Reviews in Online Social Media", IEEE, March 2017.

[2] M. Ott, C. Cardie, and J. T. Hancock. Estimating the prevalence of deception in online review communities. In ACM WWW, 2012.

[3] Li, Z. Chen, B. Liu, X. Wei, and J. Shao. Spotting fake reviews via collective PU learning. In ICDM, 2014.

[4] Nitin Jindal and Bing Liu. Opinion spam and analysis. In Proceedings of the 2008 International Conference on Web Search and Data Mining, pages 219-230. ACM, 2008.

[5] Feng, R. Banerjee and Y. Choi. Syntactic stylometry for deception detection. Proceedings of the 50th Annual Meeting of the Association for Computational Linguistics: Short Papers; ACL, 2012..

[6] N. Jindal and B. Liu. Opinion Spam and Analysis. In WSDM, 2008

[7] ]G. Wang, S. Xie, B. Liu, and P. S. Yu. Review graph based online store review spammer detection. IEEE ICDM, 2011

[8] E. D. Wahyuni and A. Djunaidy. Fake Review Detection From a Product Review Using Modied Method of Iter ative Computation Framework. In Proceeding MATEC Web of Conferences. 2016.

[9] https://www.123-reg.co.uk/blog/online-marketing/reviewspam-matter-business/ 